unerheblichen Teil des Gender Pay Gaps veranwortlich ist. Daran haben die bisher eingesetzten Instrumente nichts ändern können.

Insofern freut es mich dann doch, Sie heute hier zu unserer Veranstaltung begrüßen zu dürfen. Es freut mich deshalb, weil wir wohl kaum mehr juristischen Sachverstand zu diesem Thema finden können, als er heute hier präsent ist und weil wir in der Sache selbst engagierte Politikerinnen aller Bundestagsfraktionen hier haben, mit denen wir hoffentlich an einem Strang und den festgefahrenen Karren endlich aus dem Dreck ziehen können.

Zunächst einmal aber darf ich Sie, Frau Arenhövel, ganz besonders herzlich begrüßen. Als Thüringerin sind Sie, Frau Arenhövel, in Ihrer Landesvertretung doch zu Hause und so darf ich Ihnen zunächst dafür danken, dass wir unsere Veranstaltung in Ihrer Landesvertretung durchführen dürfen. Sie waren seit 1990 bis 2004 Mitglied des Thüringer Landtags und unter anderem Sprecherin für Soziales, Familie und Gesundheit der CDU-Landtagsfraktion. Im Thüringer Ministerium für Soziales, Familie und Gesundheit sind Sie seit dem 1. August 2004 Beauftragte für die Gleichstellung von Frau und Mann. Mit dem djb in Thüringen haben Sie u.a. zu Frau- enhäusern und zum Thüringer Gleichstellungsgesetz zusammengearbeitet und sind dort ein gern gesehener Vortragsgast. Ich freue mich ganz besonders, dass Sie sich gerade heute die Zeit genommen haben, zu uns zu kommen, denn in Thüringen findet heute der Aktionstag „Nein zu Gewalt gegen Frauen“ statt und ich bin sicher, dass Sie in Thüringen heute bei mehr als einer Veranstaltung sehr willkommen gewesen wären. Wir freuen uns jetzt alle auf Ihr Grußwort.

Prof. Dr. Marlene Schmidt ist Rechtsanwältin und zugleich Professorin für Arbeitsrecht an der Universität Frankfurt am Main. Seit ungefähr 20 Jahren arbeitet sie ausschließlich im Arbeitsrecht, im individuellen und im kollektiven. Sie berät und vertritt ausschließlich Arbeitnehmer(innen), Betriebs- und Personalräte, Gewerkschaften und Arbeitnehmerkoalitionen. Unter ihren Veröffentlichungen finden sich auch Beiträge zum Grundsatz des gleichen Entgelts für Frauen und Männer. Nicht zuletzt ist Frau Prof. Schmidt seit vielen Jahren Mitglied im djb und unserer Kommission Arbeits-, Gleichstellungs- und Wirtschaftsrecht, liebevoll auch „K1 “ genannt. Sie wurde von der Mitgliederversammlung des djb 2009 zu deren Vorsitzenden gewählt. Ich darf Sie, Dich, liebe Marlene Schmidt, jetzt um Dein Statement bitten.

\title{
Statement zum Thema Entgeltungleichheit
}

\section{Juristinnen fragen - Politikerinnen antworten, Podiumsdiskussion am 25. November 2010 in der Vertretung des Freistaats Thüringen beim Bund, Berlin}

\section{Prof. Dr. Marlene Schmidt}

Vorsitzende der Kommission Arbeits-, Gleichstellungs- und Wirtschaftsrecht des djb; Rechtsanwältin, Frankfurt am Main

Meine sehr verehrten Damen, liebe Kolleginnen,

der Deutsche Juristinnenbund bedankt sich, dass Sie sich die Zeit genommen haben, um heute hier mit uns zu diskutieren. Dank auch der Thüringischen Landesvertretung, dass wir uns heute hier in diesen schönen Räumen treffen können.

Das Thema, mit dem wir uns befassen müssen, ist allerdings ein großes Ärgernis. Denn das Grundgesetz bestimmt seit über 60 Jahren: Männer und Frauen sind gleichberechtigt. Und der EG-Vertrag besagt seit mehr als 50 Jahren: Jeder Mitgliedstaat stellt die Anwendung des Grundsatzes des gleichen Entgelts für Männer und Frauen bei gleicher oder gleichwertiger Arbeit sicher. Hierbei handelt es sich auch nicht lediglich um Verfassungslyrik oder Programmsätze, sondern um unmittelbar geltende Grundrechte, die auch im Verhältnis zwischen Privaten Geltung entfalten. Die Durchsetzung und die Verwirklichung von Entgeltgleichheit gehören daher zu den Themen, die auf der Prioritätenliste des Deutschen Juristinnenbundes ganz oben stehen.

\section{Der sogenannte „Gender Pay Gap“ und seine Ursachen}

Der Gender Pay Gap, das heißt der prozentuale Unterschied im durchschnittlichen Bruttostundenverdienst von Frauen und Männern, lag in Deutschland mit 23,2 Prozent auch im Jahr 2008 deutlich über dem Durchschnitt der Europäischen Union (18\%). Wie das Statistische Bundesamt (Destatis) anlässlich des Internationalen Frauentages am 8. März 2010 mitteilte, wiesen von den 27 Ländern der europäischen Union lediglich Estland (letzter Wert für 2007: 30,3\%), die Tschechische Republik $(26,2 \%)$, Österreich $(25,5 \%)$ und die Niederlande (letzter Wert 2007: 23,6\%) einen gegenüber Deutschland höheren geschlechtsspezifischen Verdienstabstand auf.

Dieser Verdienstabstand ist - so das Institut für Arbeitsmarkt- und Berufsforschung - teilweise auf unterschiedliche Merkmale von Frauen und Männern wie etwa das Ausbildungsniveau zurückführen, aber auch auf ungleiche Erwerbsverläufe und Charakteristika von Betrieben mit hohem Frauen- bzw. Männeranteil. Durch solche Faktoren lassen sich die festgestellten Verdienstunterschiede allerdings nicht in vollem Umfang erklären, es verbleibt ein nicht unerheblicher Rest.

So hat in Deutschland auch im Zeitraum 1993 bis 2001 ein relativ konstanter Verdienstunterschied von 12 Prozent auch zwischen solchen Frauen und Männern bestanden, die sich weder in der Ausbildung noch in Alter und Beruf unterschei- 
den und überdies im gleichen Betrieb arbeiten. ${ }^{1}$ Im internationalen Vergleich ist dies ein hoher Lohnunterschied. Bei ähnlichen Untersuchungen für die USA und Skandinavien fanden sich in so eng definierten Gruppen fast keine Lohnunterschiede zwischen Frauen und Männern mehr. ${ }^{2}$

Wir müssen also davon ausgehen: Mindestens dieser Entgeltunterschied ist auf Entgeltdiskriminierung zurückzuführen. Dabei ist diese, wie eingangs aufgezeigt wurde, schon lange untersagt.

\section{Der Anspruch auf gleiches Entgelt für gleiche und gleichwer- tige Arbeit}

\section{Das Gemeinschaftsgrundrecht auf Entgeltgleichheit}

Auf EU-Ebene ist der Grundsatz der Entgeltgleichheit von Frau und Mann in Artikel 23 Absatz 1 der EU-Grundrechtecharta, Artikel 157 VAEU und der Richtlinie 2006/54/ EG verankert.

Artikel 23 Absatz 1 der EU-Grundrechtecharta lautet:

„Die Gleichbeit von Frauen und Männern ist in allen Bereichen, einschließlich der Beschäftigung, der Arbeit und des Arbeitsentgelts, sicherzustellen."

Artikel 157 VAEU in seiner heutigen Fassung bestimmt in seinen hier maßgeblichen Absätzen 1 und 2:

„(1) Jeder Mitgliedstaat stellt die Anwendung des Grundsatzes des gleichen Entgelts für Männer und Frauen bei gleicher oder gleichwertiger Arbeit sicher.

(2) Unter „Entgelt“ im Sinne dieses Artikels sind die üblichen Grund-oder Mindestlöhne und-gehälter sowie alle sonstigen Vergütungen zu verstehen, die der Arbeitgeber aufgrund des Dienstverhältnisses dem Arbeitnehmer unmittelbar oder mittelbar in bar oder in Sachleistungen zablt.

Gleichheit des Arbeitsentgelts ohne Diskriminierung aufgrund des Geschlechts bedeutet,

a) dass das Entgelt für eine gleiche nach Akkord bezablte Arbeit aufgrund der gleichen Maßeinheit festgesetzt wird,

b) dass für eine nach Zeit bezahlte Arbeit das Entgelt bei gleichem Arbeitsplatz gleich ist. "

Und die Richtlinie 2006/54/EG bestimmt in ihrem Artikel 4:3

„Bei gleicher Arbeit oder bei einer Arbeit, die als gleichwertig anerkannt wird, wird mittelbare und unmittelbare Diskriminierung aufgrund des Geschlechts in Bezug auf sämtliche Entgeltbestandteile und -bedingungen beseitigt.

Insbesondere wenn zur Festlegung des Entgelts ein System beruflicher Einstufung verwendet wird, muss dieses System auf für männliche und weibliche Arbeitnehmer gemeinsamen Kriterien beruben und so beschaffen sein, dass Diskriminierungen aufgrund des Geschlechts ausgeschlossen werden.”

Ferner regeln die Artikel 5 bis 13 der Richtlinie 2006/54/ EG ausführlich die Anforderungen an diskriminierungsfreie Systeme der betrieblichen Altersversorgung. Damit wurde die Richtlinie 86/378/EWG und die dazu ergangene Rechtsprechung des $\mathrm{EuGH}^{4}$ kodifiziert.

Die Ungleichgewichtigkeit zwischen dem Grundsatz der Entgeltgleichheit von Frauen und Männern und den anderen
Diskriminierungsmerkmalen hat historische Ursachen: Das Gründungsmitglied Frankreich sorgte 1957 aus allein wirtschaftlichen Überlegungen für die Aufnahme dieses Grundsatzes als Artikel 119 in die Römischen Verträge. Aus Artikel 119 EWGV wurde mit Inkrafttreten des Amsterdamer Vertrags Artikel 141 EG und mit Inkrafttreten des Lissabon-Vertrags Artikel 157 VAEU.

Seit der ersten Entscheidung des EuGH zu Artikel 119 EWGV im Jahre 1971 im Fall Defrenne ${ }^{5}$ hat der Gerichtshof den nationalen Gerichten unzählige Male Auskunft über die Auslegung des Grundsatzes der Entgeltgleichheit von Frau und Mann erteilt. ${ }^{6}$ Einen nicht unerheblichen Anteil daran hatten deutsche Arbeitsrichterinnen und -richter, die seit etwa Mitte der 1980er Jahre mit Hilfe des soeben erst entdeckten Konzepts der mittelbaren Diskriminierung durch stete Vorlagen an den EuGH beharrlich auf die Beseitigung der Diskriminierung von Teilzeitbeschäftigten auch beim Arbeitsentgelt hinwirkten. ${ }^{7}$ Dennoch sind wir in Deutschland von der Realisierung der Entgeltgleichheit noch weit entfernt. Wie kann das sein?

\section{Entgeltgleichheit im AGG}

Fakt ist, dass der Grundsatz des gleichen Entgelts für gleiche und gleichwertige Arbeit in Deutschland nicht einmal mehr auf dem Papier des Gesetzbuchs existiert.

Bis zum Inkrafttreten des AGG am 18. August 2006 war der Grundsatz der Entgeltgleichheit von Frau und Mann ausdrücklich geregelt in $\$ 612$ Absatz 3 BGB, der 1980 durch das EG-rechtliche Anpassungsgesetz zur Umsetzung der Entgeltgleichheitsrichtlinie 75/117/EWG in das BGB eingefügt wurde. Die Vorschrift lautete:

„Bei einem Arbeitsverhältnis darf für gleiche oder gleichwertige Arbeit nicht wegen des Geschlechts des Arbeitnehmers eine geringere Vergütung vereinbart werden als bei einem Arbeitnehmer des anderen Geschlechts. Die Vereinbarung einer geringeren Vergütung wird nicht dadurch gerechtfertigt, dass wegen des Geschlechts des Arbeitnehmers besondere Schutzvorschriften gelten. $\int 611$ a Absatz 1 Satz 3 ist entsprechend anzuwenden."

In der juristischen Datenbank Juris finden sich für den Zeitraum der Geltung des $\mathbb{} 612$ Absatz 3 BGB (1980 bis 2006) 54 Urteile, die auf diese Norm Bezug nehmen, darunter 24 Entscheidungen des Bundesarbeitsgerichts, darunter wiederum so manche Parallelentscheidung. Mit anderen Worten:

\footnotetext{
1 IAB Kurzbericht 22/2009, 1. Im Ergebnis ebenso Gartner, Hermann/ Hinz, Thomas, Löhne von Frauen und Männern, in: IAB-Forum 1/2009, Gleichstellung - Wo Frauen und Männer in der Arbeitswelt stehen, 4 (7).

2 Gartner, Hermann/Hinz, Thomas, a.a.O.

3 Hierbei handelt es sich um die Kernvorschrift der alten Entgeltgleichheitsrichtlinie 75/117/EWG, die von der Richtlinie 2006/54/EG abgelöst wurde. Kritisch zu letzterer Kocher, Eva, Streit 2007, S. $178 \mathrm{ff}$.

4 Ausführlich hierzu Riesenhuber, Karl, 2010, § 9 Rn. 8 m.w.N.; Schmidt, Marlene, 2001, III Rn. 51-64.

5 EuGH v. 25.5.1971, Rs. 80/70, Slg. 1971, 445.

6 Vgl. nur die Aufstellung bei Schmidt, Marlene, 2001, III Rn. 48-51.

7 Sciarra, Silvana (Hrsg.), 2001.
} 
Das Bundesarbeitsgericht hatte sich durchschnittlich weniger als einmal im Jahr mit dieser Vorschrift zu befassen.

Im AGG ist der Grundsatz der Entgeltgleichheit von Frau und Mann nicht einmal mehr ausdrücklich geregelt. $\mathbb{S}$ Absatz 2 AGG bestimmt lediglich:

„Die Vereinbarung einer geringeren Vergütung für gleiche oder gleichwertige Arbeit wegen eines in \ 1 genannten Grundes wird nicht dadurch gerechtfertigt, dass wegen eines in $\mathbb{S} 1$ genannten Grundes besondere Schutzvorschriften gelten."

Damit übernimmt $\mathbb{} 8$ Absatz 2 AGG lediglich das in $\mathbb{S} 612$ Absatz 3 Satz 2 BGB a.F. enthaltene Verbot der nachteiligen Berücksichtigung von Schutzvorschriften und erweitert dieses durch den Verweis auf alle in $\mathbb{S} 1$ genannten Diskriminierungsgründe. Zugleich setzt $\mathbb{} 8$ Absatz 2 AGG die Geltung eines Verbots der Entgeltdiskriminierung wegen aller in $\mathbb{S} 1$ AGG genannten Merkmale stillschweigend voraus.

\section{Fakt ist, dass der Grundsatz des gleichen Entgelts für gleiche und gleichwertige Arbeit in Deutschland nicht einmal mehr auf dem Papier des Gesetzbuchs existiert.}

Tatsächlich ist das Verbot der Entgeltdiskriminierung heute nicht mehr ausdrücklich geregelt. Es ergibt sich mittelbar aus der Zusammenschau von $\mathbb{} 7$ Absatz 1 in Verbindung mit $\mathbb{S} 1,2$ Absatz $1 \mathrm{Nr}$. 2 AGG: Nach $\$ 7$ Absatz 1 AGG dürfen Beschäftigte nicht wegen eines in $\mathbb{1}$ genannten Grundes benachteiligt werden. $\mathrm{Zu}$ den in $\mathbb{\$} 1$ AGG genannten Gründen gehört auch das Geschlecht. Und nach $\mathbb{} 2$ Absatz 1 Nr. 2 AGG sind Benachteiligungen unter anderem wegen des Geschlechts unzulässig in Bezug auf die Beschäftigungs- und Arbeitsbedingungen einschließlich des Arbeitsentgelts.

Seit 2006 finden sich in Juris 12 Verfahren zu $\mathbb{S} 7$ Absatz 1 AGG kombiniert mit dem Stichwort „Arbeitsentgelt“, darunter erst zwei (parallele) Entscheidungen des BAG vom selben Tag zur Frage der Schichtzulagen bei Teilzeitbeschäftigung. ${ }^{8}$ Die Entscheidungsquote seit 1980 scheint sich nicht wesentlich verbessert zu haben.

\section{Der verfassungsrechtliche Auftrag zur Durchset- zung der Entgeltgleichheit}

Dieser Befund - auf der einen Hand ein Gender Pay Gap von rund 25 Prozent, auf der anderen Hand keine wirksamen rechtlichen Regelungen - ist ein Skandal, den wir nicht länger hinnehmen wollen. Denn den Gesetzgeber trifft nicht nur eine Ver- pflichtung zur Umsetzung von EG-Richtlinien, der er regelmäßig mit mehr oder minder großer Begeisterung nachkommt. Dem deutschen Gesetzgeber obliegt in punkto Entgeltgleichheit vielmehr eine verfassungsrechtliche Schutzpflicht, deren Vernachlässigung verfassungswidrig ist.

Der deutsche Gesetzgeber ist verpflichtet, die tatsächliche Gleichstellung von Frauen und Männern zu gewährleisten. Dies ergibt sich aus Artikel 3 Absatz 2 GG. Dieser lautet:

„Männer und Frauen sind gleichberechtigt. Der Staat fördert die tatsächliche Durchsetzung der Gleichberechtigung von Frauen und Männern und wirkt auf die Beseitigung bestehender Nachteile bin."

Damit stellt Artikel 3 Absatz 2 GG nach ständiger Rechtsprechung des BVerfG ein Gleichberechtigungsgebot auf, das sich auch auf die gesellschaftliche Wirklichkeit erstreckt. ${ }^{9}$ Dies ist im Rahmen der Grundgesetzänderung 1994 durch die Anfügung von Satz 2 in Artikel 3 Absatz 2 GG ausdrücklich klargestellt worden. ${ }^{10}$

Über ein bloßes Diskriminierungsverbot hinaus beauftragt Artikel 3 Absatz 2 Satz 2 GG also den Staat, zukunftsgerichtet die Gleichberechtigung der Geschlechter durchzusetzen und die Lebensverhältnisse von Frauen und Männern tatsächlich anzugleichen. Mit anderen Worten: Artikel 3 Absatz 2 GG begründet nicht nur eine staatliche Schutzpflicht vor Geschlechtsdiskriminierungen privater Dritter, ${ }^{11}$ sondern auch eine staatliche Pflicht zur Beseitigung nicht nur rechtlicher, sondern auch lediglich gesellschaftlicher Benachteiligungen. Er weist dem Staat eine gestaltende Aufgabe zu und zielt auf ein aktives staatliches Tun mittels Fördermaßnahmen. ${ }^{12}$ Diese Schutzpflicht erstreckt sich selbstverständlich auch auf das Arbeitsentgelt.

Bei der Beantwortung der Frage, wie der Gesetzgeber dem Gebot des Artikels 3 Absatz 2 GG nachkommt, steht ihm zwar ein Gestaltungsspielraum zu. ${ }^{13}$ Die Art und Weise, wie der Staat seine Verpflichtung erfüllt, die tatsächliche Durchsetzung

8 BAG v. 24.9.2008, 10 AZR 634/07, NZA 2008, 1422, sowie 638/07 und 639/07.

9 Zuletzt zum Zuschuss zum Mutterschaftsgeld, BVerfGE 109, 64 (Rn. 209) m.w.N., so beispielsweise BVerfGE 85, 191, 207; 92, 91, 109.

10 BVerfGE 109, 64 (Rn. 209); 92, 91, 109.

11 Dreier, Horst/Heun, Werner, GG, Bd. I Artikel 1-19, 2. Aufl. 2004, Art. 3 Rn. 114; Schmidt-Bleibtreu, Bruno/ Hofmann, Hans/Hopfauf, Axel (Kannengießer, Christoph), GG, 11. Aufl. 2008, Art. 3 Rn. 53, 56.

12 Jarass, Hans D./Pieroth, Bodo (Jarass), GG, 9. Aufl. 2007, Art. 3 Rn. 90; vgl. auch Dieterich/Glatzel, Mittelbar diskriminierende Steuerungswirkung des Mutterschutzes, AR-Blattei 800.2 Nr. 23.

13 Zum Gestaltungsspielraum bei Schutzpflichten allgemein BVerfGE 88, 203, 254. 
der Gleichberechtigung von Frauen und Männern zu fördern und auf die Beseitigung bestehender Nachteile hinzuwirken, obliegt seiner Ausgestaltungsbefugnis. ${ }^{14}$

Allerdings ist der Staat verfassungsrechtlich gehalten, in jedem Fall ausreichende Maßnahmen normativer und tatsächlicher Art zu ergreifen, die dazu führen, dass ein „angemessener und als solcher wirksamer Schutz" erreicht wird (sog. Untermaßverbot). ${ }^{15}$ Ist die Schutzpflichtdimension der Grundrechte betroffen, so verlangt das BVerfG von der öffentlichen Gewalt also auch vom Gesetzgeber -, dass sie bzw. er „Vorkehrungen zum Schutz des Grundrechts trifft, die nicht gänzlich ungeeignet oder völlig unzulänglich sind “. ${ }^{16}$

\section{Durchsetzungshindernisse}

Der Gender Pay Gap von knapp 25 Prozent belegt eindringlich, dass die vom Gesetzgeber bislang ergriffenen Maßnahmen zur Durchsetzung von Entgeltgleichheit völlig unzulänglich sind. Nach wie vor gibt es gravierende Hindernisse, die einer effektiven Durchsetzung der Entgeltgleichheit in Deutschland entgegenstehen:

\section{Unzureichende Transparenz in den Entlohnungssystemen}

Schon die Einforderung des Grundsatzes der Entgeltgleichheit von Frauen und Männern scheitert häufig genug daran, dass in einem Unternehmen gar nicht allgemein bekannt ist, wer genau wie viel verdient. Viele Unternehmer(innen) verpflichten ihre Arbeitnehmer(innen) ausdrücklich dazu, über ihre Einkommenshöhe Stillschweigen zu bewahren. In zwei Urteilen vom 21. Oktober $2009^{17}$ hat nun erstmals das LAG Mecklenburg-Vorpommern befunden, dass entsprechende Verschwiegenheitsklauseln im Arbeitsvertrag wegen Verstoßes gegen $\ 307$ BGB unwirksam sind, weil sie den Arbeitnehmer (gemeint ist wohl: die Arbeitnehmerin) daran hindern, Verstöße gegen den Gleichbehandlungsgrundsatz im Rahmen der Lohngestaltung gegenüber dem Arbeitgeber erfolgreich geltend zu machen. Diese Entscheidungen sind sehr zu begrüßen. Schließlich hat der EuGH bereits 1988 im Fall Danfoss ${ }^{18}$ erstmals ausgeführt, dass der erste Anschein für einen Verstoß gegen das Verbot mittelbarer Diskriminierung und die Beweislast für das Vorliegen objektiv gerechtfertigter Gründe beim (bei der) Arbeitgeber(in) liegt, sofern dem Entlohnungssystem jede Durchschaubarkeit fehlt, eine Arbeitnehmerin aber auf der Grundlage einer relativ großen Zahl von Arbeitnehmern belegen kann, dass das durchschnittliche Entgelt der weiblichen Arbeitnehmer niedriger ist als das der männlichen Arbeitnehmer. ${ }^{19}$ Selbst wenn diese Rechtsprechung bekannt wäre, setzt sie jedoch voraus, dass die Arbeitnehmerin zumindest über entsprechende statistische Angaben verfügt. Bislang sind die Unternehmen jedoch nicht verpflichtet, solche Angaben zu machen. ${ }^{20}$

\section{Unkenntnis der einschlägigen EuGH-Rechtsprechung}

Selbst wenn eine Arbeitnehmerin Kenntnis hat, dass ihr Kollege oder ihre Kollegen bei gleichen Aufgaben besser verdient als sie, herrscht in Deutschland selbst bei Arbeitsrichter(inne)n erschreckend weit verbreitete Unkenntnis über die Anforderungen der EuGH-Rechtsprechung zum Grundsatz der Entgeltgleichheit.

Üblicherweise werden Entgeltunterschiede mit vermeintlich objektiven Faktoren begründet. Aber immer dann, wenn „dem Anschein nach neutrale Vorschriften, Kriterien oder Verfahren Personen wegen eines in $\mathbb{} 1$ AGG genannten Grundes gegenüber anderen Personen in besonderer Weise benachteiligen können“, kann eine mittelbare Diskriminierung nach \3 Absatz 2 AGG gegeben sein. Vermeintlich objektive Kriterien zur Begründung von Gehaltsunterschieden, die sich in einer Vergleichsgruppe häufiger bei Männern als bei Frauen finden, sind daher immer darauf zu überprüfen, ob sie „durch ein rechtmäßiges Ziel sachlich gerechtfertigt und die Mittel zur Erreichung dieses Ziels angemessen und erforderlich“ sind.

So wird in deutschen Unternehmen zur Begründung von Entgeltunterschieden gerne auf die Dauer der Zugehörigkeit zu einem bestimmten Betrieb oder einer bestimmten Abteilung abgestellt. Hierbei handelt es sich jedoch um ein Kriterium, das Frauen gegenüber Männern im Sinne von $\mathbb{S} 3$ Absatz 2 AGG in besonderer Weise benachteiligen kann. Denn nach wie vor scheiden Frauen - im Gegensatz zu Männern - in der Familiengründungsphase häufig für längere Zeit aus dem Erwerbsleben aus. Im Allgemeinen weisen Frauen daher kürzere Betriebszugehörigkeitszeiten auf als ihre Kollegen. Folglich ist eine unterschiedliche Dauer der Betriebs- bzw. Abteilungszugehörigkeit zur Begründung unterschiedlicher Entgelthöhen nur geeignet, wenn die Anknüpfung an die Dauer der Zugehörigkeit zu einer bestimmten Abteilung zur Bestimmung der Entgelthöhe durch ein rechtmäßiges Ziel gerechtfertigt und zur Erreichung dieses Ziels geeignet und erforderlich ist. Bezüglich der Dauer der Betriebszugehörigkeit wird allgemein üblicherweise argumentiert, die mit der zunehmenden Dauer steigende Berufserfahrung befähige den (die) Arbeitnehmer(in) im Allgemeinen, seine (ihre) Arbeit besser zu verrichten. Diese Alltagsthese hält der Überprüfung am Gemeinschaftsrecht jedoch nicht stand.

14 BVerfGE 109, 64 (Rn. 210). Ein Anspruch auf bestimmte Fördermaßnahmen besteht eher selten, Jarass, Hans D./Pieroth, Bodo (Jarass), a.a.O., Rn. 91. Allerdings hat das BVerfG in E 97, 332 (348) erstmals auch eine konkrete staatliche Verpflichtung zur Schaffung von Kinderbetreuungseinrichtungen aus Art. 3 Abs. 2 GG abgeleitet. Hierauf verweisen zutreffend Dreier, Horst/Heun, Werner, a.a.O., Rn. 115.

15 Zum Verstoß gegen das Untermaßverbot durch eine (gesetzesvertretende) erkennbar ineffektive, zudem rechtlich unverbindliche Vereinbarung zwischen Wirtschaftsverbänden und Bundesregierung anstelle eines verbindlichen Gleichstellungsgesetzes vgl. Laskowski, Silke Ruth, ZRP 2001, 504.

16 BVerfGE 77, 170, 214.

172 Sa 183/og und 237/og, juris.

18 EuGH v. 17.10.1989, Rs. 109/88 (Danfoss), Slg. 1989, 3199 (Rn. 16).

19 EuGH v. 17.10.1989, Rs. 109/88 (Danfoss), Slg. 1989, 3199 (Rn. 16); v. 27.10.1993, Rs. C-127/92 (Enderby), Slg. 1993, I-5535 (Rn. 14); v. 31.5.1995, Rs. C-40o/93 (Royal Copenhagen), Slg. 1995, I-1275 (Rn. 24).

20 Eine entsprechende Verpflichtung trifft bislang allein $\S 285$ HGB für die Vergütung der Mitglieder der Geschäftsführungs- und Aufsichtsorgane von Kapitalgesellschaften. 
Mittlerweile gibt es eine ganze Reihe von EuGH-Entscheidungen zu dieser Frage. Danach kann die Dauer der Betriebs- oder Abteilungszugehörigkeit eine höhere Entlohnung nur rechtfertigen, wenn das auf diese Weise erworbene Plus an Berufserfahrung für die konkrete Tätigkeit relevant ist. ${ }^{21} \mathrm{Im}$ Fall Cad$m a n n^{22}$ stellte der Gerichtshof klar, dass es durchaus Situationen geben könne, in denen die Anknüpfung an das Kriterium des Dienstalters vom (von der) Arbeitgeber(in) im Einzelnen gerechtfertigt werden müsse. Wenn die (der) Arbeitnehmer(in) Anhaltspunkte liefere, die geeignet seien, ernstliche Zweifel daran aufkommen zu lassen, dass im vorliegenden Fall ein Rückgriff auf das Kriterium des Dienstalters zur Erreichung des genannten Zieles geeignet ist, sei es Sache des (der) Arbeitgebers (Arbeitgeberin) zu beweisen, dass (1) das Dienstalter mit der Berufserfahrung einhergehe und (2) diese den (die) Arbeitnehmer(in) befähige, seine (ihre) Arbeit besser zu verrichten, und dies auch in Bezug auf den konkreten Arbeitsplatz zutreffe.

Weithin unbekannt dürfte auch sein, dass der EuGH die Anknüpfung an das Merkmal einer bestimmten „Ausbildung“ im Sinne einer formalen beruflichen Qualifikation nur dann für nicht benachteiligend hält, wenn die Ausbildung für die Ausführung der dem (der) Arbeitnehmer(in) übertragenen Aufgaben von Bedeutung ist. ${ }^{23}$ Daher ist stets zu fragen, ob erstens die Einschätzung des (der) Arbeitgebers (Arbeitgeberin) korrekt ist und zweitens, ob die jeweiligen besonderen Kenntnisse für die Tätigkeit der (des) Betreffenden überhaupt relevant sind. Und drittens schließlich stellt sich die Frage, ob nicht auch Kolleginnen über besondere andere Kenntnisse verfügen, die honoriert werden sollten.

Noch weniger bekannt dürfte die Rechtsprechung des EuGH sein, wonach Unterschiede beim Einstellungsgehalt, die nachträglich mit der unterschiedlichen Leistungsfähigkeit der Arbeitnehmer(innen) oder der unterschiedlichen Qualität ihrer Arbeit begründet werden, regelmäßig rechtswidrig sein dürften. Denn wie der EuGH im Fall Brunnhofer ${ }^{24}$ dargelegt hat, darf ein(e) Arbeitgeber(in) in der Person des (der) Arbeitnehmers (Arbeitnehmerin) liegende Umstände, die sich bei seiner (ihrer) Einstellung nicht objektiv bestimmen lassen, sondern sich erst während der konkreten Ausübung seiner (ihrer) Tätigkeit herausstellen, wie die persönliche Leistungsfähigkeit oder die Qualität der tatsächlich erbrachten Leistungen des (der) Arbeitnehmers (Arbeitnehmerin), nicht als Rechtfertigung dafür anführen, dass für das Beschäftigungsverhältnis von Anfang an ein anderes Entgelt festgesetzt wird, als es einem (einer) Kollegen (Kollegin) des anderen Geschlechts, der (die) eine gleiche oder vergleichbare Arbeit verrichtet, gezahlt wird.

\section{Unkenntnis des Grundsatzes des gleichen Entgelts für gleichwertige Arbeit}

Ein weiteres Hindernis liegt darin, dass Deutschland völliges Entwicklungsland ist, was die Umsetzung des Grundsatzes des gleichen Entgelts für gleichwertige Arbeit anbelangt. ${ }^{25}$

Natürlich ist die Bestimmung gleichwertiger Arbeit nicht ganz einfach. ${ }^{26}$ Zusammen mit der Hans Böckler Stiftung hat die Arbeitsbewertungsexpertin Dr. Karin Tondorf jedoch ein Instrument - den sogenannten eg-check - entwickelt, mit dessen Hilfe im Betrieb oder im Unternehmen bestehende Entgeltordnungen auf Entgeltdiskriminierungen bei gleicher und gleichwertiger Arbeit untersucht werden können.

Das vom Bundesministerium für Familie, Senioren, Frauen und Jugend empfohlene Instrument „Logib-D“ vermag demgegenüber lediglich die prozentualen Entgeltunterschiede zwischen Frauen und Männern zu ermitteln, die sich bei gleicher Anzahl an Ausbildungs-, Dienstjahren und gleicher (potenzieller) Berufserfahrung sowie gleichem Anforderungsniveau und gleicher beruflicher Stellung ergeben würden. Damit kann und soll Logib-D also keineswegs Entgeltdiskriminierungen identifizieren, sondern lediglich einen Beitrag zur Ermittlung der übrigen Faktoren leisten, die dafür verantwortlich sind, dass Frauen im Schnitt 25 Prozent weniger als Männer verdienen.

Dies sollte nicht verwechselt und kann gar nicht genug betont werden. Denn auch in Unternehmen, denen Logib-D bescheinigt, dass bei ihnen alles im „grünen Bereich“ ist, sind Entgeltdiskriminierungen damit keineswegs ausgeschlossen. Da eg-check und Logib-D an verschiedenen Ursachenfaktoren des Gender Pay Gap ansetzen, können sich jedoch durchaus sinnvoll ergänzen.

\section{Widerstände der Tarifvertragsparteien}

Ein weiteres großes Hemmnis der Entgeltgleichheit sind - man muss es leider so deutlich sagen - die Tarifvertragsparteien. Diese gestalten mit ihren Tarifverträgen die Entgeltstrukturen von heute (genauer: im Jahr 2008) noch rund 75 Prozent der Arbeitnehmer(innen) in Deutschland. ${ }^{27}$ Obwohl selbstverständlich auch die Tarifvertragsparteien zur Beachtung der Entgeltgleichheit verpflichtet sind, ${ }^{28}$ liegt hier vieles noch im Argen.

Eine Entgeltordnung für den öffentlichen Dienst wird von Bund und kommunalen Arbeitgebern mit ver.di verhandelt

21 Erstmals EuGH v. 7.2.1991, Rs. C-184/89, SIg. 1991, I-297, Rn. 14, im Fall Nimz; bestätigt im Urt. v. 2.10.1997, Rs. C-1/95, Slg. 1997, I-5253, im Fall Gerster und im Urteil v. 10.3.2005, Rs. C-196/02, Slg. 2005, I-1789 (Rn. 66) im Fall Nikoloudi; zuletzt im Fall Cadman, EuGH v. 3.10.2006, Rs. C-17/05, Slg. 2006, I-9583.

22 EuGH v. 3.10.2006, Rs. C-17/05, SIg. 2006, I-9583; hierzu Schmidt, Marlene, ZESAR 2007, 86.

23 EuGH v. 17.10.1989, Rs. C-109/88 (Danfoss), Slg. 1989, I-3199 = ArbuR 1991, 120 mit Anm. Bertelsmann; v. 11.5.1999, C-309/97 (Angestelltenbetriebsrat der Wiener Gebietskrankenkasse), Slg. 1999, I-2865; BAG v. 23.2.1994, 4 AZR 219/93, BAGE 76, 44 (Rn. 51 f.).

24 EuGH v. 26.6.2001, Rs. C-381/99, Slg. 2001, I-4961 (Rn. 76 f.). In der Literatur hat diese doch enorm interessante und wichtige Entscheidung bislang keinerlei Rezeption erfahren.

25 Siehe nur die völlig berechtigte Kritik von Regine Winter an der Rechtsprechung des 4. Senats des BAG von 2005, AP Nr. 1 zu AVR Diakonisches Werk Anlage 18.

26 Zu den Arbeitsbewertungssystemen.

27 WSI-Tarifarchiv 2009: 55\% der Beschäftigten in West- und 52\% der Beschäftigten in Ostdeutschland arbeiten in Unternehmen, die der Tarifbindung unterliegen. Zusätzlich orientieren sich die Arbeitsverträge von $19 \%$ der Arbeitnehmer(innen) in West- und $23 \%$ der Arbeitnehmer(innen) in Ostdeutschland an einem Tarifvertrag.

28 Dies entspricht der Rechtsprechung des EuGH zu Art. 119 EWG das Unwirksamkeitsverdikt des $\S 7$ Abs. 2 AGG gilt daher auch für Tarifverträge. 
und soll 2011 in Kraft treten. Ver.di hat lange das Anliegen einer diskriminierungsfreien Ausgestaltung verfolgt. ${ }^{29}$ Denn Teile der alten Vergütungsordnungen im öffentlichen Dienst sind als diskriminierend bzw. als diskriminierungsverdächtig einzustufen. Nach allem, was wir gehört haben, wird ver.di diese Forderung jedoch nicht durchsetzen bzw. nicht durchsetzen können. Denn die öffentlichen Arbeitgeber(innen) sind dazu nur bereit, wenn die Summe der Entgelte dadurch nicht wächst. Mit anderen Worten: Entgeltgleichheit wäre nur zu Lasten der männlichen Kollegen durchzusetzen. Und hier kommen wir zum Kern des Problems der Entgeltgleichheit in Tarifverträgen:

Tarifverträge verkörpern quasi qua Natur der Sache einen Kompromiss, bei dem Fortschritte an einem Punkt mit Nachgeben an anderen Stellen erkauft werden müssen. Mit anderen Worten: Fortschritte bei der Entgeltgleichheit müssten mit Zugeständnissen entweder der Arbeitgeber(innen)seite oder der tarifschließenden Gewerkschaft für oftmals überwiegend männliche Mitglieder erkauft werden. Genau dies findet jedoch aus einer Vielzahl von Gründen nicht statt. ${ }^{30}$ Weiterhin darauf zu setzen, dass steter Tropfen den Stein schon noch höhlen wird, wäre nach den Erfahrungen der letzten 40 Jahre alles andere als zielführend und effektiv.

\section{Durchsetzung von Entgeltgleichheit durch Individualklage}

Dass die bestehenden Entgeltstrukturen sind, wie sie sind, ist umso problematischer und kritikwürdiger, als die gerichtliche Durchsetzung von Entgeltgleichheit der (dem) einzelnen Arbeitnehmer(in) überlassen ist. Wenn ein Unternehmen sich völlig egal ob zu Recht oder zu Unrecht - weigert, aus dem Grundsatz der Entgeltgleichheit abgeleitete Ansprüche auf ein höheres Arbeitsentgelt als das individual- oder tarifvertraglich vereinbarte Arbeitsentgelt anzuerkennen, kann es sein, dass jede(r) einzelne Arbeitnehmer(in) diesen Anspruch selbst gerichtlich durchsetzen muss. Denn das deutsche Arbeitsrecht kennt - anders als das Verbraucher- und das Umweltrecht keine Verbandsklage. Typischerweise scheuen Arbeitnehmer(innen) im bestehenden Arbeitsverhältnis aber davor zurück, ihnen zustehende Rechte gerichtlich durchzusetzen, um das Arbeitsverhältnis nicht mit einem Rechtsstreit zu belasten. Letzteres gilt selbstverständlich für Frauen wie für Männer, wirkt sich aber aufgrund der weit verbreiteten diskriminierenden Vergütungsstrukturen bei der Entgeltgleichheit besonders gravierend aus.

\section{Lösungen}

Wir meinen, die Lösungen liegen auf der Hand:

Erstens: Wir brauchen eine gesetzliche Regelung, die den Grundsatz der Entgeltgleichheit bei gleicher und gleichwertiger Arbeit regelt und vor allem unter Beachtung der Rechtsprechung des EuGH zu akzeptablen Differenzierungsbegründungen präzisiert.

Zweitens: Es muss Schluss sein mit der Geheimniskrämerei über die Verdiensthöhe. Verschwiegenheitsklauseln in Bezug auf die Höhe des Arbeitsentgelts sind für unwirksam zu erklä- ren. Gleichzeitig sollten die Unternehmen verpflichtet werden, die Entgelte, die sie ihren Arbeitnehmer(inne)n auf den verschiedenen Stufen der betrieblichen Hierarchie zahlen, in anonymisierter, aber transparenter Form offen zu legen. Überlegenswert wäre auch, einen Auskunftsanspruch gegenüber dem (der) Arbeitgeber(in) gesetzlich zu verankern.

Drittens: Geschlechtsneutrale Arbeitsbewertung sollte selbstverständlich werden. Unternehmen sollten verpflichtet werden, ihre Vergütungsstrukturen überprüfen zu lassen und gegebenenfalls anzupassen.

Viertens sollten die Tarifvertragsparteien dringend und effektiv motiviert werden, der Entgeltgleichheit zwischen Frauen und Männern erheblich größeres Gewicht als bislang beizumessen. Da Freiwilligkeit in den letzten 40 Jahren wenig gefruchtet hat, ist der Gesetzgeber dringend aufgerufen, dem ihm durch das Unionsrecht und durch Artikel 3 Absatz 2 GG erteilten Auftrag, Entgeltgleichheit zwischen Frauen und Männern effektiv zu gewährleisten, endlich nachzukommen. Modelle, wie man dies bewerkstelligen könnte, ohne zugleich unzulässig in die verfassungsrechtlich geschützte Tarifautonomie der Tarifvertragsparteien einzugreifen, existieren längst und müssten lediglich umgesetzt werden. So hat Regine Winter bereits 1994 in ihrer Dissertation Vorschläge gemacht, wie dies bewerkstelligt werden könnte. ${ }^{31}$ Und eine Gruppe von Sachverständigen unter der Leitung von Heide Pfarr hat bereits 2004 den Entwurf eines Gesetzes zur Durchsetzung der Entgeltgleichheit in kollektiven Entgeltsystemen vorgelegt, der zur Schonung der Tarifautonomie - im Wesentlichen auf eine Verfahrenslösung setzt. ${ }^{32}$

Auch der djb hat bereits 2009 im Rahmen einer Bundestagsanhörung zu dieser Frage sehr konkrete Empfehlungen vorgelegt. ${ }^{33}$

Fünftens schließlich sollte die Möglichkeit einer Verbandsklage geschaffen werden, die es im Umwelt- und Verbraucherrecht schon länger gibt.

Meine sehr verehrten Damen, Sie wissen nun, was wir von Ihnen erwarten. Sie alle sind Mitglieder des Bundestags und damit „der Gesetzgeber“. Bitte kommen Sie der Ihnen obliegenden verfassungsrechtlichen Schutzpflicht nach und verhelfen Sie der Entgeltgleichheit zur Durchsetzung. Ich freue mich auf eine anregende Diskussion.

29 Fieg, Wolfgang/Rothländer, Christian, Das ver.di-Modell einer Entgeltordnung zum TVöD und zum TV-L.

30 Deutlicher hierzu djb, Stellungnahme 2009, Fragen 35 und 36, erhältlich im Internet: 〈http://www.djb.de/Kom/K1/stog-01/> (Zugriff: 10.1.2011).

31 Winter, Regine, Frauen verdienen mehr, Zur Neubewertung von Frauenarbeit im Tarifsystem, Berlin 1994.

32 Pfarr, Heide M., FS 50 Jahre BAG, 2004, 779; zur Vereinbarkeit mit europäischem Recht siehe Kocher, Eva, KJ 2007, 22.

33 djb-Stellungnahme „Entgeltgleichheit zwischen Frauen und Männern“, Berlin, 20.1.2009, abrufbar unter <http://www.djb.de/Kom/ K1/stog-01/> (Zugriff: 10.1.2011). 